\title{
Anaphylaktische Reaktion auf Sevofluran am Arbeitsplatz
}

\author{
Anaphylactic Reaction to Sevoflurane at Work Place
}

Autoren

Institut

\author{
A. Ludwig, C. von der Helm, J. Welzel
}

Klinik für Dermatologie und Allergologie, Klinikum Augsburg
Korrespondenzadresse Prof. Dr. med. Julia Welzel Klinik für Dermatologie und Allergologie Klinikum Augsburg Sauerbruchstr. 6 86179 Augsburg julia.welzel@klinikum-augsburg. de

\section{Zusammenfassung \\ $\nabla$}

Wir berichten von einer 31-jährigen Anästhesieschwester, welche nach akzidentellem Kontakt mit Sevofluran schwere anaphylaktische Reaktionen zeigte. Wir diagnostizierten eine SevofluranSensibilisierung. Die Anästhesieschwester meidet seitdem strikt OP-Räume mit Einsatz von Sevofluran.

\section{Einleitung \\ $\nabla$}

Sevofluran ist als sicheres Inhalationsanästhetikum etabliert. Allergische Reaktionen auf diese Substanz sind bisher kaum bekannt. Es existiert ein Fallbericht einer anästhesiologischen Mitarbeiterin mit Asthmaanfällen nach erhaltener Sevofluran-Narkose sowie nach Kontakt mit Isofluran, weiterhin ein Fall einer Kontaktdermatitis eines Chirurgen auf Sevofluran [1,2]. Die allergologische Relevanz dieser Substanz bezieht sich naturgemäß nicht nur auf Patienten, welche eine entsprechende Narkose erhalten, sondern - wie in dem von uns geschilderten Fall - auf Mitarbeiter der Anästhesie mit akzidentellem Kontakt zu dieser Substanz.

\section{Kasuistik \\ $\nabla$}

Eine 31-jährige Intensivkrankenschwester in der Umschulung zur Anästhesiefachkraft stellte sich im April 2009 bei uns vor. Während der Arbeit im Aufwachraum bzw. in der urologischen Endoskopie sei es plötzlich zur Ausbildung von Urtikaria am Dekolleté und an den Unterarmen, beginnender Lippenschwellung, Schwindel, Kreislaufschwäche und Kopfschmerzen gekommen; der Blutdruck sei teilweise bis auf $50 \mathrm{mmHg}$ systolisch abgefallen. Die Gabe von Prednisolon i.v. habe zu rascher Besserung geführt. Anamnestisch ließ sich kein Auslöser eruieren. Wir dachten zu- nächst an eine Kontakturtikaria mit sekundären anaphylaktischen Kreislaufsymptomen. Allerdings stellte sich bei lediglich kurzzeitigem Betreten des Aufwachraumes eine Urtikaria an den Armen ein, was für ein volatiles Allergen sprach. Die Rücksprache mit der personalärztlichen Dienststelle und der Arbeitssicherheit ergab keine Anhaltspunkte für einen Auslöser. Danach kam es bei der Beteiligung an der Einleitung einer Maskennarkose mit kurzzeitigem Einatmen von Sevofluran erneut zur Ausbildung von Urtikaria an Unterarmen und Dekolleté mit Dyspnoe, Giemen und Blutdruckabfall auf $50 \mathrm{mmHg}$ systolisch. Es erfolgte eine sofortige antiallergische Behandlung. Während der Arbeit auf der Intensivstation, wo sie ebenso tätig war, sowie an anderen Orten waren nie allergische Symptome aufgetreten. Im Verlauf berichtete sie eine einmalig nach Kontakt mit Desfluran aufgetretene Atemnot, konnte danach aber in Räumen mit Desfluran-Narkosen problemlos arbeiten.

Ein derzeit nicht behandlungsbedürftiges allergisches Asthma bronchiale bei Sensibilisierungen auf Gräser und Schimmelpilze sei bekannt. Vor einigen Jahren sei es außerberuflich zu einer akuten generalisierten Urtikaria mit intubationspflichtigem Quincke-Ödem unklarer Genese gekommen.

Für eine Latex-Sensibilisierung gab es bei unauffälligem Pricktest auf Latex sowie negativem spezifischen IgE auf Latex keine Hinweise. Die basale Serumtryptase mit $5,5 \mu \mathrm{g} / \mathrm{ml}$ wie auch das Gesamt-IgE mit $58 \mathrm{kU} / \mathrm{l}$ waren normwertig.

Aufgrund des klaren zeitlichen Zusammenhanges äußerten wir den Verdacht auf eine Sevofluraninduzierte Anaphylaxie und führten einen einfach-blinden offenen Epikutantest mit Sevofluran in flüssiger Form durch. Hier gab die Krankenschwester kurz nach Kontakt lokal massiven Juckreiz sowie im Verlauf auch generalisierten Pruritus an; ein Erythem oder Urtikaria waren jedoch nicht sichtbar. Da sich diese Testung nur eine 
Woche nach der schweren anaphylaktischen Reaktion ereignet hatte, wiederholten wir die Hauttestung nach zwei Monaten erneut, hier ließ sich keine Reaktion auf Sevofluran sowie auf das ebenfalls getestete Desfluran, auf welches die Patientin einmalig nach akzidentellem Kontakt asthmatische Beschwerden angegeben hatte, nachweisen.

Wir empfahlen der Krankenschwester die strikte Meidung von Sevofluran und der kreuzreaktiven halogenierten Inhalationsanästhetika (z. B. Desfluran, Enfluran, Halothan) sowohl im beruflichen Umfeld als auch bei eigenen Narkosen, was bisher gelang. Die Anästhesiefachkraft wurde danach noch kurzzeitig im Rahmen ihrer Ausbildung in OP-Bereichen eingesetzt, in denen Desfluran verwendet wurde, und arbeitet seither auf einer operativen Intensivstation, auf der gelegentlich Isofluran verwendet wird, mit dem sie aber näheren Kontakt meidet. Seither hat sie keine arbeitsplatzbezogenen allergischen Beschwerden mehr. Eine eigene Operation, für die eine Vollnarkose erforderlich war, erfolgte in totaler intravenöser Anästhesie (TIVA) und wurde gut vertragen.

\section{Diskussion}

$\nabla$

Für Inhalationsnarkosen werden nach Gabe eines Einleitungshypnotikums, Relaxierung und Intubation zur Aufrechterhaltung der Narkose Inhalationsanästhetika verwendet. Zu diesen gehören volatile Anästhetika sowie die Gase Lachgas (Distickstoffmonoxid) und Xenon. Volatile Anästhetika sind flüssige Narkosemittel, die über einen Verdampfer des Narkosegerätes mit Luft und Sauerstoff gemischt verabreicht werden. Am häufigsten werden in Deutschland Sevofluran und Desfluran verwendet, seltener Enfluran oder Isofluran und kaum noch Halothan.

Die Flurane sind halogenierte Methyl-ethyl-ether. Sie enthalten alle eine Ether-Brücke. Bis auf Sevofluran riechen sie stechend und reizen die oberen Atemwege. Sevofluran ist daher auch am besten zur Narkoseeinleitung mit einer Maske geeignet.

Nach unseren Recherchen sind anaphylaktische Reaktionen auf Sevofluran und andere halogenierte Inhalationsnarkotika nur sehr selten beschrieben. Eine beruflich ausgelöste Symptomatik wurde 2006 von einer englischen Klinik publiziert, in der drei Mitarbeiter der Anästhesie Asthma, Angioödeme bzw. Hautreaktionen auf Sevofluran bzw. Isofluran entwickelt hatten [1]. Bei einer bronchialen Provokationstestung zeigten zwei der Mitarbeiter einen Abfall des FEV1 auf Isofluran, auf Sevofluran zeigten beide keine FEV1-Veränderungen. Jedoch reagierte nach der unauffälligen Sevofluran-Provokation eine Mitarbeiterin anaphylaktisch bei einer eigenen Narkose mit Sevofluran. Somit muss die Provokationstestung in diesem Fall als falsch negativ gewertet werden. Ein dritter Mitarbeiter zeigte bei bronchialer Provokation mit Sevo- und Isofluran keine Verminderung des FEV1, jedoch eine generalisierte Rötung der Haut sowie Gesichtsschwellung.

Weitere Fälle mit beruflich ausgelösten anaphylaktischen Reaktionen auf Flurane oder andere Inhalationsanästhetika konnten wir in der Literatur nicht finden, was bei der Häufigkeit ihrer Anwendung verwundert. In der Klinik in England erfolgte eine hohe Anzahl von Narkoseeinleitungen mit Fluranen, vor allem bei Kindern. Eine Absaugung war zwar vorhanden, war aber während der Einleitung der Narkose schwierig einzusetzen und war im Aufwachraum nicht vorhanden [1]. Unsere Abteilung für Arbeitssicherheit führte letzte Messungen für Sevo-, Des- und Isofluran in den OP-Sälen und der urologischen Endoskopieabteilung 2007 durch, wobei die Konzentrationen unter der analytischen Nachweisgrenze von $10 \mathrm{mg} / \mathrm{m}^{3}$ lagen, regelmäßige Messungen sind für Flurane in Deutschland nicht verpflichtend, wohl aber für Lachgas. Dieses lag deutlich unter dem Grenzwert, sodass daraus geschlossen werden kann, dass die Absaugung der Inhalationsanästhetika insgesamt bei uns effektiv ist.

Dem klinischen Allergologen stellt sich häufiger die generelle Frage nach dem Auslöser einer anaphylaktischen Reaktion, die ein Patient im Rahmen einer Intubationsnarkose erlitten hat. Mit 63\% sind die Muskelrelaxantien die häufigste Ursache, gefolgt von Latex, Injektionsanästhetika, Antibiotika, Plasmaexpandern und Opioiden [3]. Inhalationsanästhetika gelten allenfalls als sehr seltene Auslöser [4]. Da Hauttestungen wegen des volatilen Charakters außerordentlich schwierig sind, fehlt in den meisten Berichten über Zwischenfälle mit diesen Substanzen die Bestätigung einer Allergie. Am ehesten wird für z.B. Bronchospasmen bei Asthmatikern eine unspezifische Histaminliberation als Ursache angenommen $[4,5]$.

Allerdings wurden anaphylaktoide Reaktionen auf Isofluran und Enfluran sowie eine Urtikaria auf Enfluran beschrieben [4]. Weiterhin findet sich in der Literatur ein Bericht einer schweren hämodynamischen Instabilität während einer Isofluran-gestützten Narkose. Hier hatte ein 13-jähriger Patient nach Gabe von zunächst Midazolam, Fentanyl, Propofol und Atracurium anschließend Isofluran erhalten. 5 Minuten später traten eine schwere Hypotension sowie eine Tachykardie auf. Die Autoren gingen aufgrund des zeitlichen Zusammenhanges sowie der Tatsache, dass eine total intravenöse Narkose zwei Wochen später ohne Komplikationen verlief, von einer Anaphylaxie auf Sevofluran aus. Allergologische Testungen waren nicht durchgeführt worden [6]. Ähnlich berichten niederländische Kollegen von einer 7-jährigen Patientin, welche nach Gabe von Halothan und Isofluran mit einem generalisierten Erythem, Bronchospasmus sowie Tachykardie reagiert hatte. Auch hier wurden keine allergologischen Testungen durchgeführt [7].

Hauttestungen von Inhalationsanästhetika sind aufgrund ihres volatilen Charakters schwierig, da sie wegen ihres niedrigen Siedepunktes rasch verdampfen. Es können - wie in dem von uns beschriebenen Fall - offene Epikutantestungen durchgeführt werden. So wird ein Fall eines 60-jährigen Chirurgen beschrieben, welcher nach Kontakt mit Sevofluran an den exponierten Stellen an einer akuten Kontaktdermatitis litt. In einem offenen Epikutantest konnte mit einer Latenz von $24 \mathrm{~h}$ eine positive Reaktion im Sevofluran-Testfeld beobachtet werden [2]. Eine positive Hauttestung auf Sevofluran bei V.a. Soforttypreaktion ist jedoch in der Literatur nicht beschrieben.

Für den Nachweis von spezifischem IgE auf Inhalationsanästhetika stand uns kein kommerzielles Testsystem zur Verfügung. Bei den oben genannten Fällen erfolgte ebenfalls keine Bestimmung des spezifischen IgE.

Bronchiale Provokationstestungen sind, wie der eindrückliche Fall der falsch negativen Provokation aus der oben beschriebenen Publikation zeigt, ebenfalls kein ausreichend sensitives Verfahren, um eine Allergie sicher ausschließen zu können, haben aber einen hohen positiven prädiktiven Wert.

Aufgrund der wenigen beschriebenen Fälle und der Schwierigkeiten der Diagnostik können über die Kreuzallergenität der Flurane untereinander und mit anderen Inhalationsanästhetika wie zum Beispiel Halothan keine sicheren Aussagen getroffen werden. Aufgrund der ähnlichen chemischen Struktur ist sie zumindest unter den Fluranen durchaus denkbar, wie unser Fall nahelegt und die Fälle aus England [1] zeigen. 
Die von uns untersuchte Anästhesieschwester hatte eine berufsbezogene anaphylaktische Symptomatik auf Sevofluran und fraglich auch Desfluran. Sie hatte nur in Räumen, in denen diese Inhalationsanästhetika verwendet wurden, anaphylaktische Symptome und reagierte besonders heftig nach direktem Einatmen von Sevofluran, als sie bei einer Narkoseeinleitung assistierte. Wir gehen daher davon aus, dass Sevofluran der Auslöser der Anaphylaxien war. Ähnlich wie bei den Mitarbeitern der Anästhesie in England [1] im bronchialen Provokationstest zeigten sich bei unserer Patientin im Hauttest allerdings inkonstante bzw. möglicherweise falsch-negative Befunde. Wir gehen dennoch in unserem Fall aufgrund der Symptomatik am ehesten von einer IgE-vermittelten Allergie aus. Differenzialdiagnostisch ist jedoch eine nicht-IgE-vermittelte Intoleranzreaktion nicht auszuschließen.

Anästhesiemitarbeitern oder Patienten mit anaphylaktischen Reaktionen auf Inhalationsanästhetika bleibt im Fall einer eigenen bzw. erneuten OP mit erforderlicher Vollnarkose als Alternative - wie auch bei unserer Patientin - die totale intravenöse Anästhesie (TIVA).

Zusammenfassend sollten insbesondere bei Arbeitsplatz-bezogenen anaphylaktischen Ereignissen von Mitarbeitern der Anästhesie oder der Chirurgie - aber auch bei Patienten mit einer derartigen Reaktion auf eine Inhalationsnarkose - volatile Inhalationsanästhetika als Allergen in Betracht gezogen werden, auch wenn dies durch die allergologische Diagnostik nicht sicher nachgewiesen werden kann.

\section{Interessenkonflikt}

Die Autoren geben an, dass kein Interessenkonflikt besteht.
Abstract

\section{Anaphylactic Reaction to Sevoflurane at Work Place $\nabla$}

We report on a 31 year old anesthesia nurse with severe anaphylactic reactions after accidental contact with sevoflurane. We diagnosed sensitization to sevoflurane. The nurse is avoiding operation theatres with use of sevoflurane ever since.

\section{Literatur}

1 Vellore $A D$, Drought VJ, Sherwood-Jones $D$ et al. Occupational asthma and allergy to sevoflurane and isoflurane in anaesthetic staff. Allergy 2006; 61: $1485-1486$

2 Llorens Herrerias J, Delgado Navarro C, Ballester Lujan MT et al. Longterm allergic dermatitis caused by sevoflurane: a clinical report. Acta Anaesthesiol Scand 2014; 58: 1151 - 1153

3 Mertes PM, Malinovsky JM, Jouffroy L et al. Reducing the risk of anaphylaxis during anaesthesia: 2011 updated guidelines for clinical practice. J Investig Allergol Clin Immunol 2011; 21: $442-453$

4 Hobaika AB, Fernandes ML, Cancado CL et al. [Severe hemodynamic instability during the use of isoflurane in a patient with idiopathic scoliosis: case report]. Rev Bras Anestesiol 2007; 57: 177-181

5 Slegers-Karsmakers S, Stricker BH. Anaphylactic reaction to isoflurane. Anaesthesia 1988; 43: 506-507

6 Rosenberger $A D$, Treudler R, Blume-Peytavi $U$ et al. Allergien und pseudoallergische Reaktionen auf Narkosemittel. Hautarzt 1997; 49: 791 799

7 Lowry CJ, Fielden BP. Bronchospasm associated with enflurane exposure - three case reports. Anaesth Intens Care 1976; 4: 254 